\title{
AN INVERSE MAPPING THEOREM FOR SET-VALUED MAPS
}

\author{
A. L. DONTCHEV AND W. W. HAGER
}

(Communicated by James West)

\begin{abstract}
We prove that certain Lipschitz properties of the inverse $F^{-1}$ of a set-valued map $F$ are inherited by the map $(f+F)^{-1}$ when $f$ has vanishing strict derivative.
\end{abstract}

In this paper, we present an inverse mapping theorem for set-valued maps $F$ acting from a complete metric space $X$ to a linear space $Y$ with a (translation) invariant metric. We prove that, for any function $f: X \rightarrow Y$ with "vanishing strict derivative", the following properties of the inverse map $F^{-1}$ are inherited by $(f+F)^{-1}: F^{-1}$ has a closed-valued pseudo-Lipschitz selection, $F^{-1}$ is locally closed-valued and pseudo-Lipschitz, $F^{-1}$ has a Lipschitz selection, and $F^{-1}$ is locally single-valued and Lipschitz.

Let us recall that the distance from a point $x$ to a set $A$ in a metric space $(X, \rho)$ is defined by

$$
\operatorname{dist}(x, A)=\inf \{\rho(x, y): y \in A\}
$$

and the excess $e$ from the set $A$ to the set $B$ (also called the Hausdorff semidistance from $B$ to $A$ ) is given by

$$
e(B, A)=\sup \{\operatorname{dist}(x, A): x \in B\} .
$$

We denote by $B_{a}(x)$ the closed ball centered at $x$ with radius $a$. For notational convenience, $B_{\infty}(x)$ denotes $X$. Also recall that in a linear metric space $(Y, d)$ the metric $d$ is invariant if it satisfies $d(x+z, y+z)=d(x, y)$ for every $x, y, z \in Y$.

Let $F: X \rightarrow Y$ be a set-valued map. The inverse map $F^{-1}$ is defined as

$$
F^{-1}(y)=\{x \in X: y \in F(x)\},
$$

while $\operatorname{graph} F$ is the set $\{(x, y) \in X \times Y: y \in F(x)\}$. The map $F$ from a metric space $(X, \rho)$ into subsets of a metric space $Y$ is pseudo-Lipschitz

Received by the editors September 16, 1992.

1991 Mathematics Subject Classification. Primary 49K40; Secondary 26B10, 47H04, 49J52, $90 \mathrm{C} 31$.

Key words and phrases. Set-valued map, inverse function, pseudo-Lipschitz map, Lipschitz selection.

This work was supported by National Science Foundation Grant Number DMS 9022899.

The first author is on leave from the Institute of Mathematics, Bulgarian Academy of Sciences, Sofia, Bulgaria. 
around $\left(x_{0}, y_{0}\right) \in \operatorname{graph} F$ with constant $\lambda$ if there exist positive constants $\varepsilon$ and $\delta$ such that

$$
e\left(F\left(x_{1}\right) \cap B_{\varepsilon}\left(y_{0}\right), F\left(x_{2}\right)\right) \leq \lambda \rho\left(x_{1}, x_{2}\right)
$$

for every $x_{1}, x_{2} \in B_{\delta}\left(x_{0}\right)$. If $\varepsilon=+\infty$, then the map $F$ is Lipschitz in $B_{\delta}\left(x_{0}\right)$ (with respect to the Hausdorff metric). When $F$ is single-valued, this corresponds to the usual concept of Lipschitz continuity.

Pseudo-Lipschitz continuity is introduced by Aubin [1] in the context of nonsmooth analysis. Under fairly general assumptions, this property is equivalent to either openness at a linear rate for the inverse or to metric regularity [20] for the inverse (see Borwein [3], Borwein and Zhuang [4], and Penot [17]). Rockafellar [23] has shown that a closed-valued map $F$ from $\mathbf{R}^{n}$ to $\mathbf{R}^{m}$ is pseudo-Lipschitz around $\left(x_{0}, y_{0}\right)$ if and only if the function " $(x, y) \rightarrow$ the distance from $y$ to $F(x)$ " is Lipschitz in a neighborhood of $\left(x_{0}, y_{0}\right)$. Although in this paper we do not use nonsmooth analysis, our results are closely related to research in this area; see Clarke [5], Ioffe [10, 11], and Mordukhovich $[13,14]$.

In the sequel, we denote by $(\mathrm{L} i), i=1,2,3,4$, the following properties for an arbitrary map $A$ from $X$ to the subsets of $Y$ :

(L1) $A$ has a closed-valued pseudo-Lipschitz selection around $\left(x_{0}, y_{0}\right)$. That is, for some given $\left(x_{0}, y_{0}\right) \in \operatorname{graph} A$, there exist a set-valued map $S: X \rightarrow Y$ and a constant $\beta>0$ such that $y_{0} \in S\left(x_{0}\right)$, the set $S(x)$ is a closed subset of $A(x)$ for $x \in B_{\beta}\left(x_{0}\right)$, and $S$ is pseudo-Lipschitz around $\left(x_{0}, y_{0}\right)$.

(L2) $A$ is locally closed-valued and pseudo-Lipschitz around $\left(x_{0}, y_{0}\right)$. That is, for some given $\left(x_{0}, y_{0}\right) \in \operatorname{graph} A$, there exist $\theta>0$ and $\beta>0$ such that the set $A(x) \cap B_{\theta}\left(y_{0}\right)$ is closed, for $x \in B_{\beta}\left(x_{0}\right)$, and the map $x \rightarrow A(x) \cap B_{\theta}\left(y_{0}\right)$ is pseudo-Lipschitz around $\left(x_{0}, y_{0}\right)$.

(L3) $A$ has a Lipschitz selection around $\left(x_{0}, y_{0}\right)$. That is, for some given $\left(x_{0}, y_{0}\right) \in \operatorname{graph} A$, there exist a single-valued map $s: X \rightarrow Y$ and a constant $\beta>0$ such that $y_{0}=s\left(x_{0}\right), s(x) \in A(x)$ for $x \in B_{\beta}\left(x_{0}\right)$, and $s$ is Lipschitz in $B_{\beta}\left(x_{0}\right)$.

(L4) $A$ is locally single-valued and Lipschitz around $\left(x_{0}, y_{0}\right)$. That is, for some given $\left(x_{0}, y_{0}\right) \in \operatorname{graph} A$, there exist $\theta>0$ and $\beta>0$ such that the map $x \rightarrow A(x) \cap B_{\theta}\left(y_{0}\right)$ is single-valued and Lipschitz in $B_{\beta}\left(x_{0}\right)$.

It is clear that (Li) implies (L1), for $i=2,3,4$, and (L4) implies (L3).

Let us recall that a (single-valued) function $f$ from a normed linear space $X$ into a normed linear space $Y$ is strictly differentiable at $x_{0} \in X$ if there exists a linear and continuous operator from $X$ to $Y$, denoted $\nabla f\left(x_{0}\right)$, with the property that, for every $\varepsilon>0$, there exists $\delta>0$ such that

$$
\left\|f\left(x_{1}\right)-f\left(x_{2}\right)-\nabla f\left(x_{0}\right)\left(x_{1}-x_{2}\right)\right\| \leq \varepsilon\left\|x_{1}-x_{2}\right\|
$$

whenever $\left\|x_{i}-x_{0}\right\|<\delta, i=1,2$. (For a thorough discussion of this concept see Nijenhuis [16].) In this paper, functions with vanishing strict derivative play an important role. We say that a (single-valued) function $f$ from a metric space $(X, \rho)$ into a metric space $(Y, d)$ is strictly stationary at $x_{0} \in X$ if, for every $\varepsilon>0$, there exists $\delta>0$ such that

$$
d\left(f\left(x_{1}\right), f\left(x_{2}\right)\right) \leq \varepsilon \rho\left(x_{1}, x_{2}\right)
$$

whenever $\rho\left(x_{i}, x_{0}\right)<\delta, i=1,2$.

In this paper we prove 
Theorem (inverse mapping). Let $F$ be a set-valued map from a complete metric space $X$ to subsets of a linear space $Y$ with an invariant metric, let $y_{0} \in F\left(x_{0}\right)$, and let $f: X \rightarrow Y$ be a (single-valued) function which is strictly stationary at $x_{0}$. Then, for $i=1,2,3,4$, the following are equivalent.

(i) The map $F^{-1}$ has the property $(\mathrm{L} i)$ around $\left(y_{0}, x_{0}\right)$.

(ii) The map $(f+F)^{-1}$ has the property $(\mathrm{L} i)$ around $\left(y_{0}+f\left(x_{0}\right), x_{0}\right)$.

The proof of the theorem is based on

Lemma (fixed point). Let $(X, \rho)$ be a complete metric space; let $\Phi$ map $X$ to the closed subsets of $X$; let $\xi_{0} \in X$; and let $r$ and $\lambda$ be such that, $0 \leq \lambda<1$,

(a) $\operatorname{dist}\left(\xi_{0}, \Phi\left(\xi_{0}\right)\right)<r(1-\lambda)$,

(b) $e\left(\Phi\left(x_{1}\right) \cap B_{r}\left(\xi_{0}\right), \Phi\left(x_{2}\right)\right) \leq \lambda \rho\left(x_{1}, x_{2}\right)$ for all $x_{1}, x_{2} \in B_{r}\left(\xi_{0}\right)$.

Then $\Phi$ has a fixed point in $B_{r}\left(\xi_{0}\right)$; that is, there exists $x \in B_{r}\left(\xi_{0}\right)$ such that $x \in \Phi(x)$. If $\Phi$ is single-valued, then $x$ is the unique fixed point of $\Phi$ in $B_{r}\left(\xi_{0}\right)$.

A proof of the lemma will be presented after the proof of the theorem.

Proof of Theorem. We begin with the implication (ii) $\Rightarrow$ (i) for the case (L1). In this case, there exists a set-valued map $\Psi$ with the following properties: $x_{0} \in \Psi\left(y_{0}+f\left(x_{0}\right)\right)$; there exists a positive constant $\beta$ such that $\Psi(y)$ is a closed subset of $(f+F)^{-1}(y)$ for every $y \in B_{\beta}\left(f\left(x_{0}\right)+y_{0}\right)$; and, for some positive constants $\alpha$ and $\gamma$,

$$
e\left(\Psi\left(y_{1}\right) \cap B_{\alpha}\left(x_{0}\right), \Psi\left(y_{2}\right)\right) \leq \gamma d\left(y_{1}, y_{2}\right)
$$

whenever $y_{1}, y_{2} \in B_{\beta}\left(y_{0}+f\left(x_{0}\right)\right)$. Take an arbitrary $\gamma^{+}>\gamma$, and let $\varepsilon>0$ be such that

$$
\gamma \varepsilon<1 \text { and } \gamma<(1-\gamma \varepsilon) \gamma^{+} .
$$

Since $f$ is strictly stationary at $x_{0}$, we can choose $\alpha$ smaller, if necessary, so that

$$
d\left(f\left(x_{1}\right), f\left(x_{2}\right)\right) \leq \varepsilon \rho\left(x_{1}, x_{2}\right)
$$

for every $x_{1}, x_{2} \in B_{\alpha}\left(x_{0}\right)$. Now choose $a$ and $b$ such that

$$
0<a \leq \min \{\alpha, \beta / 4 \varepsilon\}, \quad 0<b<\min \left\{\beta / 2, a / 4 \gamma^{+}\right\} .
$$

Given $x \in B_{a}\left(x_{0}\right)$ and $y \in B_{b}\left(y_{0}\right),(3)$ and (4) imply that

$$
\begin{aligned}
d\left(y+f(x), y_{0}+f\left(x_{0}\right)\right) & \leq d\left(y, y_{0}\right)+d\left(f(x), f\left(x_{0}\right)\right) \\
& \leq b+\varepsilon a<\beta / 2+\beta / 4<\beta .
\end{aligned}
$$

Hence $y+f(x) \in B_{\beta}\left(y_{0}+f\left(x_{0}\right)\right)$ and $\Psi(y+f(x))$ is a closed subset of $(f+F)^{-1}(y+f(x))$ whenever $x \in B_{a}\left(x_{0}\right)$ and $y \in B_{b}\left(y_{0}\right)$.

Let $\Phi$ be the map defined by $\Phi(x, y)=\Psi(y+f(x))$. If $x \in \Phi(x, y)$ for some $x \in B_{a}\left(x_{0}\right)$ and $y \in B_{b}\left(y_{0}\right)$, then $x \in \Psi(y+f(x)) \subset(f+F)^{-1}(y+f(x))$, which implies that $x \in F^{-1}(y)$. If $\Gamma(y)$ denotes the set of fixed points of $\Phi(\cdot, y)$ in $B_{a}\left(x_{0}\right)$, we will show that $\Gamma$ is a closed-valued pseudo-Lipschitz selection of $F^{-1}$ around $\left(y_{0}, x_{0}\right)$. Clearly $x_{0} \in \Gamma\left(y_{0}\right)$; we have already observed that $\Gamma(y) \subset F^{-1}(y)$ for every $y \in B_{b}\left(y_{0}\right)$. It remains to be shown that $\Gamma(y)$ is closed, for any $y \in B_{b}\left(y_{0}\right)$, and $\Gamma$ is pseudo-Lipschitz around $\left(x_{0}, y_{0}\right)$. 
To prove that $\Gamma(y)$ is closed for any $y \in B_{b}\left(y_{0}\right)$, we suppose that $x_{k} \in \Gamma(y)$ and $x_{k} \rightarrow x$ as $k \rightarrow \infty$. Since $f$ is Lipschitz in $B_{a}\left(x_{0}\right)$ with Lipschitz constant $\varepsilon$ by (3), we have

$$
\begin{aligned}
\operatorname{dist}\left(x_{k}, \Phi(x, y)\right) & \leq e\left(\Psi\left(y+f\left(x_{k}\right)\right) \cap B_{a}\left(x_{0}\right), \Psi(y+f(x))\right) \\
& \leq \gamma d\left(f\left(x_{k}\right), f(x)\right) \leq \gamma \varepsilon \rho\left(x_{k}, x\right) \rightarrow 0
\end{aligned}
$$

as $k \rightarrow \infty$. Since $\Phi(x, y)$ is closed, we conclude that $x \in \Gamma(y)$.

To prove that the map $\Gamma$ is pseudo-Lipschitz around $\left(y_{0}, x_{0}\right) \in \operatorname{graph} \Gamma$, we take any $y^{\prime}, y^{\prime \prime} \in B_{b}\left(y_{0}\right), y^{\prime} \neq y^{\prime \prime}$, and we show that, for every $x^{\prime} \in$ $\Gamma\left(y^{\prime}\right) \cap B_{a / 2}\left(x_{0}\right)$, one can find $x^{\prime \prime} \in \Gamma\left(y^{\prime \prime}\right)$ such that

$$
\rho\left(x^{\prime}, x^{\prime \prime}\right) \leq \gamma^{+} d\left(y^{\prime}, y^{\prime \prime}\right) .
$$

This is accomplished by proving that the map $\Phi\left(\cdot, y^{\prime \prime}\right)$ has a fixed point in the closed ball with center $x^{\prime}$ and radius $l=\gamma^{+} d\left(y^{\prime}, y^{\prime \prime}\right)$. In order to apply the lemma, we first observe that

$$
\begin{aligned}
\operatorname{dist}\left(x^{\prime}, \Phi\left(x^{\prime}, y^{\prime \prime}\right)\right) & \leq e\left(\Phi\left(x^{\prime}, y^{\prime}\right) \cap B_{a}\left(x_{0}\right), \Phi\left(x^{\prime}, y^{\prime \prime}\right)\right) \\
& =e\left(\Psi\left(y^{\prime}+f\left(x^{\prime}\right)\right) \cap B_{a}\left(x_{0}\right), \Psi\left(y^{\prime \prime}+f\left(x^{\prime}\right)\right)\right) \\
& \leq \gamma d\left(y^{\prime}, y^{\prime \prime}\right)<l(1-\gamma \varepsilon) .
\end{aligned}
$$

Since $l<a / 2$ by (4), (1) and (3) imply that, for each $x_{1}, x_{2} \in B_{l}\left(x^{\prime}\right)$, we have

$$
\begin{aligned}
e(\Phi & \left.\left(x_{1}, y^{\prime \prime}\right) \cap B_{l}\left(x^{\prime}\right), \Phi\left(x_{2}, y^{\prime \prime}\right)\right) \\
& \leq e\left(\Phi\left(x_{1}, y^{\prime \prime}\right) \cap B_{a}\left(x_{0}\right), \Phi\left(x_{2}, y^{\prime \prime}\right)\right) \\
& =e\left(\Psi\left(y^{\prime \prime}+f\left(x_{1}\right)\right) \cap B_{a}\left(x_{0}\right), \Psi\left(y^{\prime \prime}+f\left(x_{2}\right)\right)\right) \\
& \leq \gamma d\left(f\left(x_{1}\right), f\left(x_{2}\right)\right) \leq \gamma \varepsilon \rho\left(x_{1}, x_{2}\right) .
\end{aligned}
$$

The fixed point lemma with $\xi_{0}=x^{\prime}, \lambda=\gamma \varepsilon$, and $r=l$ yields the existence of $x^{\prime \prime} \in \Gamma\left(y^{\prime \prime}\right)$ satisfying (5). This completes the proof of case (L1).

The proof of (ii) $\Rightarrow$ (i) in cases (L2)-(L4) can be deduced from the proof of (L1). In case (L2) there exist $\theta \in(0,+\infty]$ and $\beta>0$ such that the set $(f+F)^{-1}(y) \cap B_{\theta}\left(x_{0}\right)$ is closed, for every $y \in B_{\beta}\left(f\left(x_{0}\right)+y_{0}\right)$, and the map $\Psi$ defined by $\Psi(y)=(f+F)^{-1}(y) \cap B_{\theta}\left(x_{0}\right)$ is pseudo-Lipschitz around $\left(f\left(x_{0}\right)+y_{0}, x_{0}\right)$. By repeating the proof of case (L1) with $a$ and $b$ as in (4) and $a \leq \theta$, we obtain that if $\Gamma(y)$ is the set of fixed points of $\Phi(\cdot, y)=\Psi(y+f(\cdot))$ in $B_{a}\left(x_{0}\right)$, then $\Gamma(y)$ is closed for every $y \in B_{b}\left(y_{0}\right)$ and pseudo-Lipschitz around $\left(y_{0}, x_{0}\right)$. It can be verified that, for any $y \in B_{b}\left(y_{0}\right), x \in \Gamma(y)$ if and only if $x \in F^{-1}(y) \cap B_{a}\left(x_{0}\right)$. Hence, the map $y \rightarrow F^{-1}(y) \cap B_{a}\left(x_{0}\right)$ is closed-valued and pseudo-Lipschitz around $\left(y_{0}, x_{0}\right)$.

Now suppose that $(f+F)^{-1}$ has a single-valued Lipschitz selection $\Psi$ around $\left(y_{0}+f\left(x_{0}\right), x_{0}\right)$ with constant $\gamma$; that is, $\Psi(y) \in(f+F)^{-1}(y)$, for every $y \in B_{\beta}\left(f\left(x_{0}\right)+y_{0}\right)$, and $\Psi$ is Lipschitz continuous in $B_{\beta}\left(f\left(x_{0}\right)+y_{0}\right)$ with constant $\gamma$. Choose $\gamma^{+}$and $\varepsilon$ as in (2) and $\alpha>0$ such that (3) holds for each $x_{1}, x_{2} \in B_{\alpha}\left(x_{0}\right)$. Let $a$ and $b$ be as in (4), let $r=\gamma^{+} b$ and $\lambda=\gamma \varepsilon$, and let $\Phi(x, y)=\Psi(y+f(x))$. If $y \in B_{b}\left(y_{0}\right)$, then

$$
\begin{aligned}
\rho\left(x_{0}, \Phi\left(x_{0}, y\right)\right) & =\rho\left(\Psi\left(y_{0}+f\left(x_{0}\right)\right), \Psi\left(y+f\left(x_{0}\right)\right)\right) \\
& \leq \gamma d\left(y, y_{0}\right) \leq \gamma b<r(1-\lambda)
\end{aligned}
$$


and

$$
\begin{aligned}
\rho\left(\Phi\left(x_{1}, y\right), \Phi\left(x_{2}, y\right)\right) & =\rho\left(\Psi\left(y+f\left(x_{1}\right)\right), \Psi\left(y+f\left(x_{2}\right)\right)\right) \\
& \leq \gamma d\left(f\left(x_{1}\right), f\left(x_{2}\right)\right) \leq \lambda \rho\left(x_{1}, x_{2}\right)
\end{aligned}
$$

whenever $x_{1}, x_{2} \in B_{r}\left(x_{0}\right)$. The fixed point lemma implies that, for every $y \in B_{b}\left(y_{0}\right)$, there exists a unique fixed point $x(y)$ of $\Psi(y+f(\cdot))$ in $B_{r}\left(x_{0}\right)$. Since $\Psi(y+f(x(y))) \in F^{-1}(y), x(\cdot)$ is a single-valued selection of $F^{-1}$ in $B_{b}\left(y_{0}\right)$. For each $y^{\prime}, y^{\prime \prime} \in B_{b}\left(y_{0}\right)$, we have

$$
\begin{aligned}
\rho\left(x\left(y^{\prime}\right), x\left(y^{\prime \prime}\right)\right) & =\rho\left(\Psi\left(y^{\prime}+f\left(x\left(y^{\prime}\right)\right)\right), \Psi\left(y^{\prime \prime}+f\left(x\left(y^{\prime \prime}\right)\right)\right)\right) \\
& \leq \gamma d\left(y^{\prime}, y^{\prime \prime}\right)+\gamma d\left(f\left(x\left(y^{\prime}\right)\right), f\left(x\left(y^{\prime \prime}\right)\right)\right) \\
& \leq \gamma d\left(y^{\prime}, y^{\prime \prime}\right)+\gamma \varepsilon \rho\left(x\left(y^{\prime}\right), x\left(y^{\prime \prime}\right)\right)
\end{aligned}
$$

which implies that

$$
\rho\left(x\left(y^{\prime}\right), x\left(y^{\prime \prime}\right)\right) \leq \gamma^{+} d\left(y^{\prime}, y^{\prime \prime}\right) .
$$

Thus $x(\cdot)$ is a Lipschitz selection of $F^{-1}$ around $\left(y_{0}, x_{0}\right)$, and case (L3) is established.

Finally, suppose that $\Psi(y)=(f+F)^{-1}(y) \cap B_{\theta}\left(x_{0}\right)$ is single-valued and Lipschitz near $y_{0}$ for some $\theta>0$. Choosing $a$ and $b$ as in (4) and $a<\theta$ and repeating the argument used in case (L3), we obtain that, for $r=\gamma^{+} b$, there exists a unique fixed point $x(y)$ of $\Psi(y+f(\cdot))$ in $B_{r}\left(x_{0}\right)$, for any $y \in B_{b}\left(y_{0}\right)$, and $x(\cdot)$ is Lipschitz continuous on $B_{b}\left(y_{0}\right)$. Since $r<a$, it follows that $x(y)=F^{-1}(y) \cap B_{r}\left(x_{0}\right)$. Hence, $F^{-1}$ is locally single-valued and Lipschitz around $\left(y^{0}, x^{0}\right)$.

To show that $(\mathrm{i}) \Rightarrow$ (ii), let $f$ be an arbitrary (single-valued) function which is strictly stationary at $x_{0}$, and let $F$ have the property $(\mathrm{L} i)$ around $\left(y_{0}, x_{0}\right)$. Then $(-f+f+F)^{-1}$ has the property $(\mathrm{L} i)$ around $\left(-f\left(x_{0}\right)+f\left(x_{0}\right)+y_{0}, x_{0}\right)$ and $-f$ is strictly stationary at $x_{0}$. From the first part of the proof, we conclude that the map $(f+F)^{-1}$ has the property $(\mathrm{L} i)$ around $\left(f\left(x_{0}\right)+y_{0}, x_{0}\right)$. This completes the proof of the theorem.

Remark. From the proof we can estimate the Lipschitz constant; if $(f+F)^{-1}$ has the property $(\mathrm{L} i)$ around $\left(y_{0}+f\left(x_{0}\right), x_{0}\right), i=1,2,3,4$, with constant $\gamma$, then, for any $\gamma^{+}>\gamma, F^{-1}$ has property $(\mathrm{L} i)$ around $\left(y_{0}, x_{0}\right)$ with constant $\gamma^{+}$.

Proof of Lemma. We employ the standard iterative procedure for contracting mappings. However, compared with the known fixed point theorem for setvalued mappings (see, e.g., $[9,15,18]$ ), we use the excess rather than the Hausdorff distance.

By assumption (a), there exists $\xi_{1} \in \Phi\left(\xi_{0}\right)$ such that $\rho\left(\xi_{1}, \xi_{0}\right)<r(1-\lambda)$. Proceeding by induction, suppose that there exists $\xi_{k+1} \in \Phi\left(\xi_{k}\right) \cap B_{r}\left(\xi_{0}\right)$ for $k=1,2, \ldots, n-1$ with

$$
\rho\left(\xi_{k+1}, \xi_{k}\right)<r(1-\lambda) \lambda^{k}
$$

By assumption (b),

$$
\operatorname{dist}\left(\xi_{n}, \Phi\left(\xi_{n}\right)\right) \leq e\left(\Phi\left(\xi_{n-1}\right) \cap B_{r}\left(\xi_{0}\right), \Phi\left(\xi_{n}\right)\right) \leq \lambda \rho\left(\xi_{n}, \xi_{n-1}\right)<r(1-\lambda) \lambda^{n}
$$

This implies that there exists $\xi_{n+1} \in \Phi\left(\xi_{n}\right)$ such that

$$
\rho\left(\xi_{n+1}, \xi_{n}\right)<r(1-\lambda) \lambda^{n} \text {. }
$$


By the triangle inequality,

$$
\rho\left(\xi_{n+1}, \xi_{0}\right) \leq \sum_{k=0}^{n} \rho\left(\xi_{k+1}, \xi_{k}\right)<r(1-\lambda) \sum_{k=0}^{n} \lambda^{k}<r .
$$

Hence, $\xi_{n+1} \in \Phi\left(\xi_{k}\right) \cap B_{r}\left(\xi_{0}\right)$ and the induction step is complete.

For $n>m$, we have

$$
\rho\left(\xi_{n}, \xi_{m}\right) \leq \sum_{k=m}^{n-1} \rho\left(\xi_{k+1}, \xi_{k}\right)<r(1-\lambda) \sum_{k=m}^{n-1} \lambda^{k}<r \lambda^{m}
$$

Thus $\xi_{k}$ is a Cauchy sequence converging to some $\xi \in B_{r}\left(\xi_{0}\right)$. By assumption (b),

$$
\operatorname{dist}\left(\xi_{n}, \Phi(\xi)\right) \leq e\left(\Phi\left(\xi_{n-1}\right) \cap B_{r}\left(\xi_{0}\right), \Phi(\xi)\right) \leq \lambda \rho\left(\xi, \xi_{n-1}\right)
$$

The triangle inequality implies that

$$
\operatorname{dist}(\xi, \Phi(\xi)) \leq \rho\left(\xi, \xi_{n}\right)+\operatorname{dist}\left(\xi_{n}, \Phi(\xi)\right) \leq \rho\left(\xi_{n}, \xi\right)+\lambda \rho\left(\xi_{n-1}, \xi\right),
$$

which approaches zero as $n$ increases. Since $\Phi(\xi)$ is closed, we conclude that $\xi \in \Phi(\xi)$. If $\Phi$ is single-valued, assumption (b) implies that $\xi$ is the unique fixed point of $\Phi$ in $B_{r}\left(\xi_{0}\right)$. This completes the proof.

We note that our paper [6] contains a prototype of the fixed point lemma for maps acting in Banach spaces.

We present some corollaries of the inverse mapping theorem related to known results.

Corollary 1. If $X$ is a complete metric space, $Y$ is a linear space with an invariant metric, and the single-valued functions $\phi: X \rightarrow Y$ and $\psi: X \rightarrow Y$ have the property that the difference $\phi-\psi$ is strictly stationary at $x_{0}$, then the inverse map $\phi^{-1}$ possesses property $(\mathrm{L} i)$ around $\left(\phi\left(x_{0}\right), x_{0}\right)$ if and only if $\psi^{-1}$ possesses property $(\mathrm{L} i)$ around $\left(\psi\left(x_{0}\right), x_{0}\right)$.

Proof. Take $F=\phi$ and $f=\psi-\phi$, and apply the theorem.

From case (L2) of the inverse mapping theorem we obtain

Corollary 2. Let $X$ be a complete metric space, let $Y$ be a linear space with an invariant metric, and let $F: X \rightarrow Y$ be a set-valued map with closed graph. If $y_{0} \in F\left(x_{0}\right)$ and $f: X \rightarrow Y$ is a continuous function which is strictly stationary at $x_{0}$, then the following are equivalent.

(i) The map $F^{-1}$ is pseudo-Lipschitz around $\left(y_{0}, x_{0}\right)$.

(ii) The map $(f+F)^{-1}$ is pseudo-Lipschitz around $\left(y_{0}+f\left(x_{0}\right), x_{0}\right)$.

Proof. To prove that (ii) $\Rightarrow(\mathrm{i})$, first observe that the map $\Psi(\cdot)=(f+F)^{-1}(\cdot)$ is closed-valued and pseudo-Lipschitz around $\left(y_{0}+f\left(x_{0}\right), x_{0}\right)$. From case (L2) of the theorem with $\theta=+\infty$, there exists $\tau>0$ such that the map $y \rightarrow$ $F^{-1}(y) \cap B_{\tau}\left(x_{0}\right)$ is pseudo-Lipschitz around $\left(y_{0}, x_{0}\right)$; hence, $F^{-1}$ is pseudoLipschitz around $\left(y_{0}, x_{0}\right)$. The implication (ii) $\Rightarrow$ (i) applied to the map $-f+(f+F)$ yields (i) $\Rightarrow$ (ii), which completes the proof.

For maps acting in Euclidean spaces, Corollary 2 can be deduced using tools from nonsmooth analysis, as shown in [13]. For $F=0$ this corollary implies 
Corollary 3. Let $X$ and $Y$ be Banach spaces, and let $\phi: X \rightarrow Y$ be a continuous function which is strictly differentiable at $x_{0}$. Then the inverse map $\phi^{-1}$ is pseudo-Lipschitz around $\left(\phi\left(x_{0}\right), x_{0}\right)$ if and only if $\nabla \phi\left(x_{0}\right)$ is surjective.

Proof. Taking $f(x)=\phi(x)-\nabla \phi\left(x_{0}\right) x$ and $F(x)=\nabla \phi\left(x_{0}\right) x$ and applying Corollary 2, we conclude that $\phi^{-1}$ is pseudo-Lipschitz around $\left(\phi\left(x_{0}\right), x_{0}\right)$ if and only if the map $y \rightarrow \nabla \phi\left(x_{0}\right)^{-1} y$ is pseudo-Lipschitz around $\left(\nabla \phi\left(x_{0}\right) x_{0}, x_{0}\right)$. From the open mapping principle, $\nabla \phi\left(x_{0}\right)^{-1}$ is pseudo-Lipschitz if and only if $\nabla \phi\left(x_{0}\right)$ is surjective.

The "if" part of Corollary 3 resembles the classical Graves theorem [8] (see Aubin and Frankowska [2, p. 95]), taking into account that openness at a linear rate of $\phi$ is equivalent to the pseudo-Lipschitz property of the inverse $\phi^{-1}$. If $\nabla \phi\left(x_{0}\right)$ is surjective and one-to-one, then $\phi^{-1}$ is locally single-valued and strictly differentiable at $\phi\left(x_{0}\right)$ and the strict derivative of $\phi^{-1}$ at $\phi\left(x_{0}\right)$ is $\nabla \phi\left(x_{0}\right)^{-1}$ (see Leach [12]).

The Robinson-Ursescu theorem (see [18, 19, 20, 24]) can be equivalently formulated as follows: Let $X$ and $Y$ be Banach spaces, let $G: X \rightarrow Y$ have closed and convex graph, and let $\left(x_{0}, y_{0}\right) \in \operatorname{graph} G$. Then the map $G^{-1}$ is pseudo-Lipschitz around $\left(y_{0}, x_{0}\right)$ if and only if $y_{0} \in$ core $G(X)$. Hence, Corollary 2 implies the following result which is more general than Corollary 3.

Corollary 4. Let $X$ and $Y$ be Banach spaces, let $\phi: X \rightarrow Y$ be a continuous function which is strictly differentiable at $x_{0}$, and suppose that the set-valued map $G: X \rightarrow Y$ has convex and closed graph with $y_{0} \in \phi\left(x_{0}\right)+G\left(x_{0}\right)$. Then the following are equivalent.

(i) $y_{0}$ lies in the core of the image of $\left[\phi\left(x_{0}\right)+\nabla \phi\left(x_{0}\right)\left(\cdot-x_{0}\right)+G(\cdot)\right]$.

(ii) The map $(\phi+G)^{-1}$ is pseudo-Lipschitz around $\left(y_{0}, x_{0}\right)$.

Proof. Combine the Robinson-Ursescu theorem with Corollary 2, identifying $f(x)$ with $\phi(x)-\phi\left(x_{0}\right)-\nabla \phi\left(x_{0}\right)\left(x-x_{0}\right)$ and $F(x)$ with $\phi\left(x_{0}\right)+\nabla \phi\left(x_{0}\right)$ $\cdot\left(x-x_{0}\right)+G(x)$.

Note that the implication (i) $\Rightarrow$ (ii) can be essentially extracted from results in [3,17, 18]. For maps $G$ having graphs of the form $C \times K$, where $C$ is a closed convex set in a Banach space and $K$ is a closed and convex cone in a Banach space, the equivalence of (i) and (ii) (in a metric regularity form) is established in [20]. Another characterization of the surjectivity property of the strict derivative can be obtained from case (L3) of Corollary 1.

Corollary 5. Let $X$ be a Banach space, let $Y$ be a linear space with an invariant metric, and let $\phi: X \rightarrow Y$ be a function which is strictly differentiable at $x_{0}$. If the inverse map $\phi^{-1}$ has a Lipschitz selection around $\left(\phi\left(x_{0}\right), x_{0}\right)$, then $\nabla \phi\left(x_{0}\right)$ is surjective. The converse holds if $X$ is a Hilbert space.

Proof. Suppose that $\phi^{-1}$ has a Lipschitz selection around $\left(\phi\left(x_{0}\right), x_{0}\right)$. By Corollary 1 , case (L3), with $\psi(x)=\nabla \phi\left(x_{0}\right) x, \nabla \phi\left(x_{0}\right)^{-1}$ has a Lipschitz selection around $\left(\nabla \phi\left(x_{0}\right) x_{0}, x_{0}\right)$. Thus $\nabla \phi\left(x_{0}\right)^{-1} y \neq \varnothing$ for $y$ near $y_{0}:=\nabla \phi\left(x_{0}\right) x_{0}$ and $\nabla \phi\left(x_{0}\right)$ is surjective. Conversely, if $X$ is a Hilbert space, $\nabla \phi\left(x_{0}\right)$ is surjective, and $P$ denotes the pseudo-inverse of $\nabla \phi\left(x_{0}\right)$, then the map $y \rightarrow$ $y_{0}+P\left(y-y_{0}\right)$ is a Lipschitz selection of $\nabla \phi\left(x_{0}\right)^{-1}$ around $\left(y_{0}, x_{0}\right)$. By Corollary 1 , case (L3), $\phi^{-1}$ has a Lipschitz selection around $\left(\phi\left(x_{0}\right), x_{0}\right)$. 
Our final application illuminates the main conclusion of this paper. Under appropriate assumption, we can replace the original inverse function problem by another, perhaps simpler, inverse function problem. We apply case (L4) of the inverse mapping theorem to the following variational inequality: Find $x \in X$ such that

$$
y \in \phi(x)+\partial \Omega(x),
$$

where $\phi: X \rightarrow X^{*}, X$ is a Banach space, $X^{*}$ is its dual, $\Omega$ is a set in $X$, $y \in X^{*}$ is a parameter, and $\partial \Omega(x)$ is the normal cone to the set $\Omega$ at the point $x$; that is,

$$
\partial \Omega(x)= \begin{cases}\left\{v \in X^{*}:\langle v, z-x\rangle \leq 0 \text { for each } z \in \Omega\right\} & \text { if } x \in \Omega, \\ \varnothing & \text { if } x \notin \Omega .\end{cases}
$$

Suppose that $x_{0}$ is a solution of (6) for $y=0$ and $\phi$ is strictly differentiable at $x_{0}$. By the inverse mapping theorem, Lipschitz properties of the (set of) solutions of (6) depending on $y$ are equivalent to corresponding Lipschitz. properties for solutions of the linearized variational inequality: Find $x \in X$ such that

$$
y \in T(x), \quad \text { where } T(x):=\phi\left(x_{0}\right)+\nabla \phi\left(x_{0}\right)\left(x-x_{0}\right)+\partial \Omega(x) .
$$

Robinson [21] introduced the following concept: The variational inequality (6) is strongly regular at $\left(x_{0}, 0\right)$ if there exist neighborhoods $\mathscr{U}$ of the origin in $X^{*}$ and $\mathscr{V}$ of $x_{0}$ such that the map $y \rightarrow T^{-1}(y) \cap \mathscr{V}$ is single-valued and Lipschitz in $\mathscr{U}$. By considering a variational inequality depending nonlinearly on the parameter $y$, he proved that strong regularity implies the existence of a locally unique solution which is Lipschitz in $y$. For an extension of this result to nonsmooth functions, see [22]. By case (L4) of the inverse mapping theorem, strong regularity is necessary and sufficient for Lipschitz stability of the solution to the variational inequality (6). More precisely, we have

Corollary 6. The following are equivalent.

(i) The variational inequality (6) is strongly regular at $\left(x_{0}, 0\right)$.

(ii) There exist neighborhoods $V$ of $x_{0}$ and $U$ of 0 and a Lipschitz function $x(\cdot): U \rightarrow V$ such that, for all $y \in U, x(y)$ is the unique solution of (6) in $V$.

Proof. Take $f(x)=\phi(x)-\phi\left(x_{0}\right)-\nabla \phi\left(x_{0}\right)\left(x-x_{0}\right)$ and $F(x)=T(x)$, and apply case (L4) of the theorem.

Related results for maps defined implicitly by variational inequalities and optimization problems are developed in [7].

\section{REFERENCES}

1. J.-P. Aubin, Lipschitz behavior of solutions to convex minimization problems, Math. Oper. Res. 9 (1984), 87-111.

2. J.-P. Aubin and H. Frankowska, Set-valued analysis, Birkhäuser, Boston, MA, 1990.

3. J. M. Borwein, Stability and regular points of inequality systems, J. Optim. Theory Appl. 48 (1986), 9-52.

4. J. M. Borwein and D. M. Zhuang, Verifiable necessary and sufficient conditions for openness and regularity of set-valued maps, J. Math. Anal. Appl. 134 (1988), 441-459. 
5. F. H. Clarke, Optimization and nonsmooth analysis, Wiley, New York, 1983.

6. A. L. Dontchev and W. W. Hager, Lipschitz stability in nonlinear control and optimization, SIAM J. Control Optim. 31 (1993), 569-603.

7. __ Implicit functions, Lipschitz maps and stability in optimization, Math. Oper. Res. (to appear).

8. L. M. Graves, Some mapping theorems, Duke Math. J. 17 (1950), 111-114.

9. A. D. Ioffe and V. M. Tikhomirov, Theory of extremal problems, North-Holland, Amsterdam, 1979.

10. A. D. Ioffe, Regular points of Lipschitz functions, Trans. Amer. Math. Soc. 251 (1987), 61-69.

11. _ Global surjection and global inverse mapping theorems in Banach spaces, Ann. New York Acad. Sci., vol. 491, New York Acad. Sci., New York, 1987, pp. 181-189.

12. E. B. Leach, A note on inverse function theorem, Proc. Amer. Math. Soc. 12 (1961), 694-697.

13. B. Mordukhovich, Complete characterization of openness, metric regularity, and Lipschitzian properties of multifunctions, Trans. Amer. Math. Soc. 340 (1993), 1-35.

14. __ Stability theory for parametric generalized equations and variational inequalities via nonsmooth analysis, IMA Preprints Series 994, 1992.

15. S. B. Nadler, Jr., Multi-valued contraction mappings, Pacific J. Math. 30 (1969), 475-488.

16. A. Nijenhuis, Strong derivatives and inverse mappings, Amer. Math. Monthly 81 (1974), 969-980.

17. J.-P. Penot, Metric regularity, openness and Lipschitz multifunctions, Nonlinear Anal. 13 (1989), 629-643.

18. S. M. Robinson, An inverse-function theorem for a class of multivalued functions, Proc. Amer. Math. Soc. 41 (1973), 211-218.

19. $\_$Regularity and stability for convex multivalued functions, Math. Oper. Res. 1 (1976), 130-143.

20. _ Stability theory for systems of inequalities, Part II: Differentiable nonlinear systems, SIAM J. Numer. Anal. 13 (1976), 497-513.

21. _ Strongly regular generalized equations, Math. Oper. Res. 5 (1980), 43-62.

22. __ An implicit-function theorem for a class of nonsmooth functions, Math. Oper. Res. 16 (1991), 292-309.

23. R. T. Rockafellar, Lipschitz properties of multifunctions, Nonlinear Anal. 9 (1985), 867-885.

24. C. Ursescu, Multifunctions with closed convex graphs, Czechoslovak Math. J. 25 (1975), 438-441.

Mathematical Reviews, 416 Fourth Street, Ann Arbor, Michigan 48107

E-mail address: ald@math.ams.org

Department of Mathematics, University of Florida, Gainesville, Florida 32611

E-mail address: hager@math.uf $1 . e d u$ 